\title{
Amyloidogenic Processing of APP in Lipid Rafts
}

\author{
Madepalli K. Lakshmana*, Subhojit Roy, Kaihong Mi and David E. Kang \\ Department of Neurosciences, University of California, San Diego, 9500 Gilman Drive, La Jolla, CA 92093, USA
}

\begin{abstract}
Increased generation of amyloid $\beta$ peptide $(A \beta)$ derived from amyloid precursor protein (APP) is the primary pathological characteristic of Alzheimer's disease (AD). However, the sub cellular compartment in which APP undergoes cleavage by secretases to generate $A \beta$ is not precisely known. Compelling evidences suggest that amyloidogenic processing of APP occurs in lipid rafts. An indirect support for lipid raft processing of APP includes the localization of A $\beta$, APP C-terminal fragments (CTFs), APP holoprotein and secretases in the lipid raft microdomains, although few studies failed to find APP in the lipid rafts. The indirect support also comes from both experimental and clinical studies involving modulation of cholesterol levels and its effect on $\mathrm{A} \beta$ generation. Moderate depletion of cholesterol results in significant reduction in $A \beta$ levels and increased dietary intake of cholesterol leads to higher levels of $A \beta$ production suggesting that amyloidogenic processing of APP strongly depends on cholesterol levels and therefore on lipid raft integrity. More convincing evidence that lipid rafts are critical for amyloidogenic processing of APP comes from studies using antibody-mediated co-patching of APP and BACE1 which results in lipid raft association of APP and BACE1 and increased $A \beta$ generation. Further, an endosome/lipid raft targeting of $\beta$-secretase inhibitor by sterol-mediated anchoring leading to reduced $A \beta$ generation also suggests that lipid rafts are pivotal for amyloidogenic processing of APP. In the absence of an effective therapy for AD, proteins responsible for delivery of APP to lipid rafts including LRP, RanBP9 and ApoER2 may be excellent therapeutic targets in AD.
\end{abstract}

Keywords: Amyloid precursor protein, A $\beta$, lipid rafts, C-terminal fragments, BACE1, $\gamma$-secretase, amyloidogenic processing, RanBP9, LRP, detergent resistant membranes.

\section{INTRODUCTION}

Alzheimer's disease (AD) is a devastating neurodegenerative disorder of the elderly characterized by progressive memory loss and impairment of cognition along with changes in personality and behavior. It was recently reported that nearly 5.3 million Americans are affected by AD requiring about 148 billion dollars in annual costs, and a new case of $\mathrm{AD}$ is diagnosed every seventy seconds [1]. $\mathrm{AD}$ is also the sixth leading cause of death and the second most feared disease after cancer. Recent statistics have also revealed that while death rate due to stroke, heart disease and cancer has a decreasing trend, death related to Alzheimer's disease has actually been increased by $47 \%$ between 2000 and 2006. In most developed countries, life expectancy has gradually increased and now humans live longer and longer. But unfortunately age is the single most consistent risk factor for $\mathrm{AD}$ and longer life invariably leads to the increased likelihood of AD diagnosis. The presence of extracellular amyloid plaques and intracellular neurofibrillary tangles, the two major neuropathological hallmarks of $\mathrm{AD}$ are still the diagnostic features of $\mathrm{AD}$, ever since the first description by Alois Alzheimer of his patient, Auguste D [2, 3]. Amyloid plaques are parenchymal deposits of amyloid $\beta$ protein $(A \beta)$, a fibrillous $4-\mathrm{kDa}$ protein of 38 to 43 amino acids derived from amyloid precursor protein (APP) by the sequential actions of $\beta$ - and $\gamma$ - secretases. Neurofibrillary tangles are neuronal inclusions composed of paired helical filaments of the hyperphosphorylated protein tau. As of today, there is no

*Address correspondence to this author at the Dept. of Neurosciences, MC0691, UC San Diego, 9500 Gilman Drive, La Jolla, CA 92093, USA; Tel: 858-822-1025; Fax: 858-822-1021; E-mail: mlakshmana@ucsd.edu effective therapy for $\mathrm{AD}$ and the available treatments can neither slow nor reverse the disease progression as they are not designed to treat the underlying cause of $\mathrm{AD}$. To develop effective therapy, it is important to understand, how $A \beta$ is generated, what sub cellular compartments produce $A \beta$ and what factors modulate generation of $A \beta$.

\section{APP Biology and Processing}

APP is a single spanning type I integral transmembrane protein ubiquitously expressed in all tissues. APP belongs to a conserved group of proteins comprising APP-like protein 1 (APLP1) and 2 (APLP2), the Drosophila APPL and the C. elegans $A P L-1$, which all complicates the functional analysis of $A P P$ [4]. When APP was discovered more than two decades ago [5], it was initially thought that it could be a receptor. Although in recent years many extracellular proteins and intracellular adaptor proteins have been shown to bind to APP, it is still not clear whether APP can function as a bona fide receptor and a true ligand has not yet been convincingly demonstrated. The precise function of APP family of proteins is not known. To understand the role of these proteins, null mice for single or combination of APP genes have been generated by gene knock out techniques. Homozygous APP-null mice are viable and fertile, but mice showed $15-20 \%$ reduced body weight and decreased locomotor activity. About $70 \%$ of mice also exhibited reactive gliosis suggesting impaired neuronal function [6]. Single disruption of $A P L P 1$ or $A P L P 2$ also caused only minor phenotypes [7, 8]. A combination of $A P L P 2-/-/ A P P-/-$ or $A P L P 2-/-/ A P L P 1-/-$ gene knock out resulted in early postnatal lethal phenotype suggesting functional redundancy among the members [8]. Disruption of APLP1-/-/APP-/- 
double genes resulted in viable mice [8]. Mice with the disruption of all three APP genes die shortly after birth due to cranial abnormalities resulting in cortical dysplasia implying an essential role for the members of APP family in normal brain development. However, only APP contains the encoding sequence for $\mathrm{A} \beta$, and produces the $\mathrm{AD}$-associated A $\beta$ peptide.

The heterogeneity of APP arises by alternative splicing of APP gene resulting in the production of three major isoforms of 695, 751 and 770 amino acids with a molecular mass ranging from 110 and $140 \mathrm{kDa}$ [9]. The complexity also arises from a variety of posttranslational modifications such as phosphorylation, the addition of $\mathrm{N}$ - and O-linked sugars, and sulfation [10-12]. The difference between 695 versus 751 and 770 isoforms are the presence of 56 amino acid motif that is analogous to the Kunitz-type serine protease inhibitors [KPI] in the 751 and 770 isoforms suggesting that these two forms have an additional function. Both 751 and 770 forms are highly expressed in both neuronal and non neuronal cell types, while 695 isoform is expressed almost exclusively in neurons [13].

The vast majority of APP is constitutively cleaved by $\alpha-$ secretase (ADAM10/TACE/ADAM17) in the middle of $A \beta$ sequence at 17 amino acids $\mathrm{NH} 2$ - terminal to the single transmembrane domain of APP, thereby precluding the formation of intact $\mathrm{A} \beta[14,15]$. This non-amyloidogenic pathway results in the release of the large soluble ectodomain fragment $(\mathrm{sAPP} \alpha)$, while 83-amino acid Cterminal fragment $(\mathrm{CTF} \alpha)$ is retained in the membrane. Alternatively, a small proportion of APP is cleaved in the amyloidogenic pathway, leading to the secretion of $A \beta$ peptides (38 to 43 amino acids) via two proteolytic enzyme activities, $\beta$ - and $\gamma$-secretase, now known as BACE1 and presenilin complexes, respectively [16. 17]. In the amyloidogenic pathway, APP is first cut by $\beta$-secretase resulting in the release of a soluble APP $\beta$ (sAPP $\beta$ ) and the membrane retains 99 amino acid $\mathrm{CTF}(\mathrm{CTF} \beta)$. Both $\mathrm{C} 83$ and $\mathrm{C} 99$ are substrates of $\gamma$-secretase. Upon cleavage by $\gamma$-secretase, C83 gives rise to P3 fragment and APP intracellular domain (AICD). C99 on the other hand leads to the generation of $A \beta$. The endocytic processing of wild type APP from the cell surface is apparently required for $A \beta$ generation by delivering APP to intracellular sites enriched in $\beta$ - and $\gamma$ secretase activities [18, 19]. In contrast, the Swedish APP mutation is cleaved by BACE1 on the way to the cell surface and does not require endocytosis for $A \beta$ generation. In either case, the proteolytic processing of APP to generate A $\beta$ requires the trafficking of APP such that APP and BACE1 are brought together in close proximity for $\beta$-secretase cleavage to occur. The molecular basis by which these steps are regulated is complex and incompletely understood.

\section{The Concept of Lipid Rafts}

The idea that functional lipid rafts (LRs) exist in the cell membranes was introduced by Simons and Ikonen in 1997 [20]. According to their 'raft hypothesis', sphingolipid and cholesterol-rich membrane microdomains act as platforms bringing together functionally related proteins for numerous cellular functions from membrane trafficking to cell adhesion and signal transduction. The basis for raft hypothesis is the differential biophysical properties of glycosphingolipids and sphingomyelins which require a higher temperature for gel to liquid transition than do sphingolipids. This hypothesis postulates that lateral assembly of sphingolipids and cholesterol creates rafts floating in a glycerophospholipid-rich environment, recruiting specific membrane proteins while excluding others. Thus, lipid rafts are defined as liquidordered (lo), detergent-resistant cholesterol and sphingolipidrich microdomains proposed to exist within cellular membranes. Biochemically, the lipid rafts are characterized by their insolubility in detergents such as Triton X-100 (TX100 ) or CHAPS at $4^{\circ} \mathrm{C}$, forming what are called as detergent-insoluble glycolipid-enriched complexes (DIGs) [21, 22], also called as detergent-resistant membranes (DRM). However, concrete data on abundance, size and number of DIGs in each cell or number of proteins in each DIG in neurons is not precisely known. In fibroblasts, individual rafts may be between $10-100 \mathrm{~nm}$ in diameter, corresponding to $\sim 3,500$ sphingolipid molecules and about 10-30 proteins. Multiple evidences suggest that glycosyl-phosphatidylinositol (GPI) anchored proteins, transmembrane proteins and doubly acylated proteins tend to cluster in these microdomains [23-25]. Many other proteins also can move in and out of DIGs during signal transductions. Most studies on lipid rafts are conducted after biochemical isolation by sucrose-gradient ultracentrifugation in cold after extraction with either TX-100 or CHAPS [26, 27]. However, since rafts are considered to be too small to be resolved by conventional microscopy techniques [28], a direct visualization of these dynamic raft structures on the native membranes has not been convincingly demonstrated [29]. With technological advancement in the areas of microscopy and/or imaging such as fluorescence resonance energy transfer (FRET) and total internal reflection fluorescence microscopy (TIRFM), it may be possible to validate the concept of lipid rafts in near future.

\section{APP Processing in Lipid Rafts}

In the last two decades enormous progress has been made on how $\mathrm{A} \beta$ is produced in terms of its precursor protein, and the enzymes involved in its generation. It is now clear that $\mathrm{A} \beta$ is produced by the consecutive actions of $\beta$ - and $\gamma$-secretases, which releases A $\beta$ from its precursor protein, APP [16, 17]. An alternative cleavage by $\alpha$-secretase at the $17^{\text {th }}$ amino acid of $A \beta$ sequence prevents the generation of intact $A \beta$ $[14,15]$. But precisely in which intracellular compart-ment APP undergoes $\alpha, \beta$ and $\gamma$-secretase cleavages is not fully understood. There are conflicting results about whether $A \beta$ is generated in the secretory pathway such as endop-lasmic reticulum, ERGIC, Golgi and trans-Golgi network (TGN) or in the endocytic pathway including plasma mem-brane, or early and late endosomes/lysosomes. Although open to debate, it is generally believed that $A \beta$ is mainly generated in the endosomes as APP is trafficked through endocytosis from the plasma membrane $[18,19]$. All these intracellular compartments have been demonstrated to contain lipid rafts. The half-life of APP is less than an hour and studies in cell cultures have shown that only about $10 \%$ APP is present at the cell surface and that $70 \%$ of them are internalized within minutes. After endocytosis, a fraction of APP may be recycled back to the plasma membrane, but considerable amount are subjected to degradation in lysosomes. 
Initially, the suspicion that amyloidogenic processing of APP occurs in lipid rafts were derived from the observation that considerable amount of $\mathrm{A} \beta$ is present in lipid rafts [30, 31 ] and indirectly also on the basis of localization of $\beta$ - and $\gamma$-secretase enzymes in lipid rafts. Consistent with these results, several independent studies found the presence of both $\beta$ - and $\gamma$-secretase components such as BACE $[32,33]$, presenilin proteins [34-36], presenilin activity [37] and completely assembled and biologically active components of $\gamma$-secretase such as nicastrin, aph-1 and pen-2 [38, 39] in lipid rafts. Thus not only $A \beta$, but also the complete $A \beta$ generating machinery has been shown to be present within the rafts. These observations have led to the conclusion that the principal sites of $A \beta$ production are the specialized cholesterol rich lipid rafts.

\section{a) Evidences from Cholesterol Modulation}

The indirect evidence for the amyloidogenic processing of APP in lipid rafts also comes from numerous studies on the influence of cholesterol on $A \beta$ generation and the incidence of $\mathrm{AD}$. As the core component of lipid rafts is cholesterol, which maintains the lipid raft integrity, any change in the levels of cholesterol and its associated effect on APP processing and $A \beta$ generation is interpreted to represent the effect of lipid rafts. The best genetic risk factor for AD demonstrated so far by genetic association studies is APOE $\varepsilon 4$ allele [40, 41]. As ApoE4 is responsible for cholesterol homeostasis in the brain, it is suggested that cholesterol may play an important role in $\mathrm{A} \beta$ generation. In $\mathrm{CHO}$ cells, depletion of membrane cholesterol prevents $\gamma$-secretase cleavage of APP and replacement of cholesterol restored $\gamma$ secretase cleavage suggesting that $\gamma$-secretase activity is cholesterol dependent [36]. Moreover, it is well known from epidemiological studies that patients taking statins, the inhibitors of 3-hydroxy-3-methylglutaryl-CoA (HMA-CoA) reductase, a rate limiting enzyme in cholesterol biosynthesis show reduced incidence of $\mathrm{AD}[42,43]$, likely due to reduced synthesis of cholesterol [44, 45]. There is also epidemiological evidence that increased cholesterol levels in mid-life increases the likelihood of developing AD in later life [46]. There are multiple other evidences supporting critical role for cholesterol in A $\beta$ generation. Animal models also support role of statins in reducing cholesterol levels and amyloid plaque burden. For instance, widely used statins such as simvastatin and lovastatin given at high doses to guinea pigs for three weeks reduced both $A \beta 40$ and $A \beta 42$ levels in both the cerebrospinal fluid (CSF) and brain homogenates [47]. In another study, two-month treatment of an animal model of Alzheimer's disease expressing APP751 with London (V717I) and Swedish (K670M/N671L) mutations by an inhibitor of an enzyme responsible for conversion of cholesterol to cholesteryl-esters (ACAT), led to reduction of soluble $A \beta$ which was followed by a dramatic reduction in the number of plaques [48]. Surprisingly, spatial learning behavior was also improved in these animals. The dependency of A $\beta$ generation by cholesterol and the presence of secretases in DIGs were interpreted to suggest that amyloidogenic processing of APP occurs in lipid rafts.

\section{b) Contradictory Reports on the Role of Cholesterol}

However, the role of cholesterol and its associated DIGs in $\mathrm{A} \beta$ generation is complicated by contradictory reports from both clinical and experimental studies. The foremost contradiction is the demonstration of the inability of statins including lovastatin, simvastatin and pravastatin to reduce cholesterol levels in both primary neuronal cultures and in vivo in guinea pigs $[47,49]$. Therefore, cholesterol-independent pathway for statin mediated amyloidogenic processing of APP has also been suggested. In a recent Multi-Institutional Research in Alzheimer's Genetic Epidemiology trial (MIRAGE study), statin therapy for at least six months reduced the incidence of $\mathrm{AD}$, but non-statin cholesterollowering drugs failed to influence the incidence of $\mathrm{AD}$, suggesting that the mechanism of action of statins in modulating $A \beta$ production is cholesterol-independent [50]. This is consistent with another study showing reduced levels of membrane cholesterol in the hippocampus of AD patients [51]. In another in vivo study, a transgenic mouse model of AD expressing APP with Swedish mutation were fed high cholesterol diet leading to increased cholesterol levels in the serum and brain. Surprisingly, both $A \beta$ and sAPP levels were inversely correlated with serum and brain cholesterol levels, without any appreciable changes in the levels of APP holoprotein or CTFs [52], contradicting the results from many other studies. Such inconsistent experimental and clinical results may be partly because of the differences in age of the experimental animals, gender, and duration of exposure to the cholesterol lowering drug and so on. In fact, a study by Park et al. [53] treated both male and female twelve-month old Tg2576 APP transgenic mice, with lovastatin for three weeks and measured $A \beta$ levels. Lovastatin treatment led to prompt reduction of serum cholesterol levels in both males and females, but unexpectedly, $A \beta$ generation and plaque deposition was enhanced only in females but not in males, without any alterations in the levels of full-length APP, CTFs or PS1 suggesting gender specific effects of cholesterol lowering drugs.

\section{c) Evidences from Endocytosis}

Such inconsistent results on amyloidogenic processing of APP and A $\beta$ generation by cholesterol lowering drugs is also likely because of their direct effect on endocytosis of APP [54]. In this study lovastatin treatment reduced the levels of full-length APP and $\beta$-secretase activity in low density lipid rafts (LDLR), a non-classical lipid raft, characterized by its insolubility in Lubrol WX. Almost half of APP co-localized with the lipid raft marker flotillin in the Lubrol WX insoluble membrane fractions prepared from hippocampus, but none in the TX-100 insoluble microdomains, suggesting that lovastatin lowers amyloidogenic processing of APP not by depleting cholesterol in the typical TX-100 insoluble lipid rafts, but by influencing the distribution of APP in the LDLR microdomains. The authors' argument that lovastatin reduced amyloidogenic processing of APP by inhibiting APP endocytosis is consistent with the idea that endosomes are the major sites for $\beta$-cleavage as reflected by a positive correlation between endocytic activity and $\beta$-cleavage of APP [18, 55-60]. Koo and Squazzo [18] inhibited internalization of APP as inferred from the uptake of radio iodinated 1G7 Fab fragment by two different approaches, first by the deletion of cytoplasmic tail within APP and second by depletion of potassium. In multiple experiments, inhibition of endocytosis by either method consistently reduced generation of $A \beta$. Conversely, over expression of Rab5, a positive 
regulator of endocytosis in the fibroblast like cells stably expressing APP695 led to 2.5-fold increase in the levels of both $A \beta 40$ and $A \beta 42$ in the medium, followed by a 2 -fold increase in the levels of $\beta$ CTFs and sAPP $\beta$ with respect to sAPP $\alpha$ [59]. Thus, inhibition of endocytosis reduces, while activation increases the amyloidogenic processing of APP. A positive correlation between APP endocytosis and A $\beta$ generation is also supported indirectly by the localization of $\beta$-secretase in the Golgi and endosomes and that $\beta$-secretase has optimal activity at acidic $\mathrm{pH}$ found in the endosomes $[61,62]$. If APP remains in the cell surface, it is likely cleaved by $\alpha$-secretase and if it is internalized in to endosomes, it is most likely cleaved by $\beta$-secretase followed by $\gamma$-secretase thereby generating $\mathrm{A} \beta$.

\section{d) Evidences from Antibody Co-Patching Experiments}

The idea that amyloidogenic processing of APP occurs in the lipid rafts was first proposed by Ehehalt and colleagues in 2003 [63]. Initially, they showed that $A \beta$ production in N2a cells is critically dependent on cholesterol levels. Lovastatin treatment of $\mathrm{N} 2 \mathrm{a}$ cells decreased cholesterol levels by $85 \%$ which totally abolished $\mathrm{A} \beta$ generation followed by a clear decrease in the levels of $\beta \mathrm{CTF}$, indicating that BACE cleavage of APP is reduced. Based on the knowledge that antibodies could crosslink raft and non-raft markers into distinct patches [64, 65], next in an elegant experiment, involving co-patching of APP and BACE1 by antibody cross-linking at the cell surface, Ehehalt and colleagues showed increased generation of $A \beta$ in a cholesterol and endocytosis-dependent manner. They used GPI-anchored protein placental alkaline phosphatase (PLAP) as a raft marker and endocytosis defective transferrin receptor (TfR del 5-41) with the deletion of the sorting signal, cytosolic aa 5-41 as a non-raft marker. Quantitative analysis revealed that the percentage of both APP and BACE1 in LRs was significantly more after antibody cross linking than before. After cross linking both APP and BACE1 colocalized with each other and with the raft marker, PLAP, suggesting that APP and BACE1 are associated with lipid rafts. The authors also demonstrated that partitioning of APP and BACE1 into the lipid rafts alone is insufficient for efficient amyloidogenic processing of APP, but also depends on endocytosis. To demonstrate this, endocytosis was inhibited by expressing RN-tre, a Rab5 GTPase activating protein, which inhibits clathrin-dependent endocytosis [66] and also by expressing a dynamin mutant K44A, which affects both clathrin-dependent and independent endocytosis [67]. Inhibition of endocytosis by both methods significantly reduced $A \beta$ generation, implying that amyloidogenic processing of APP requires internalization. A schematic diagram illustrates how $\mathrm{A} \beta$ generation depends not only on clustering of individual lipid rafts containing APP and BACE1but also endocytosis (Fig. 1).

\section{e) Evidences from Localization of APP and its Derivatives}

Both $\beta$ and $\gamma$-secretases are localized to TX-100insoluble microdomains within specific cellular compartments [32-39], but how these secretases gain access to its substrate APP is unknown. If amyloidogenic processing of APP is to occur in lipid rafts as proposed above, then APP must be localized within the rafts. Many studies have been conducted to localize APP in the rafts with conflicting



Fig. (1). A schematic diagram of plasma membrane illustrating distribution of raft and non-raft membrane microdomains. Rafts are distinguished from non-rafts by its enrichment in cholesterol ( $30 \%)$ and sphingolipids (10-15\%). Available evidence indicates that majority of APP is associated with non-raft microdomains while $\beta$ - and $\gamma$-secretases are associated with both raft and non-rafts. As the number of protein molecules in each raft is limited, APP and secretases are unlikely to be localized in the same raft. Following endocytosis, small individual rafts are clustered together bringing APP and secretases in close proximity leading to amyloidogenic processing of APP and A $\beta$ generation (B). However, if BACE1 containing raft is not clustered together with APP containing raft, endocytosis need not lead to amyloidogenic processing of APP (A). 
results. In one of the earliest studies, a small fraction of APP was shown to be co-localized with glypiated glycoprotein, F3, a GPI-anchored protein characteristic of lipid raft proteins in TX-100 insoluble light density complexes prepared from forebrain neuronal cultures and fractionated by sucrose gradient velocity centrifugation [68]. However in another study [69] using similar conditions including the detergent, TX-100 and sucrose gradient ultracentrifugation, membranes prepared from mouse cerebellum as well as SH-SY5Y neuroblastoma cells contained many GPI-anchored proteins such as alkaline phosphatase, 5' -nucleotidase and F3 protein, but not APP. In another study [44], rat hippocampal neurons were infected with recombinant Semliki Forest Virus (SFV) carrying APP expressing construct and TX-100-resistant membranes were fractionated by sucrose step-gradient ultra centrifugation. A fraction of cellular APP was found to float in the low density fractions corresponding to lipid rafts [44]. In the same study it was also shown that lovastatin mediated cholesterol depletion greatly reduced lipid raft association of APP. Further, based on results obtained from pulse-chase experiments, the authors also suggested that newly synthesized APP is unlikely to be associated with lipid rafts but presumably only after APP enters Golgi, similar to many GPI-anchored proteins [21]. In COS7 cells a significant amount of APP detected by $22 \mathrm{C} 11$ antibody was shown to be co-associated with caveolin-enriched membrane fractions [70]. Similar results were also obtained in HEK293 and MDCK cells. Because the antibody 22C11 detects not only APP but also APLPs, to confirm the specificity of APP association with caveolin-enriched membranes, a recombinant approach was used. A recombinant HA-tagged APP695 and myc-tagged caveolin-1 were transiently transfected into COS7 cells and detected by their corresponding antibodies. The results showed that majority of recombinant APP695 and caveolin-1 were again co-fractionated and co-localized within caveolae-enriched membrane microdomains. Similar to lipid rafts, caveolar membrane domains also has highly concentrated cholesterol and sphingolipids. Although this study suggested a predominant $\alpha$-cleavage of APP in these domains, in W4 rat neuroblastoma cells over expression of caveolin-1 decreased secretion of sAPP $\alpha$ in a dose-dependent manner, while the levels of sAPP $\beta$ were significantly increased suggesting that in caveolae also $\beta$-secretase processing of APP can occur [71], although neuronal DIGs do not have detectable levels of caveolin-1.

A significant amount of APP and both $\alpha \mathrm{CTF}$ and $\beta \mathrm{CTF}$ along with $A \beta 40$ and $A \beta 42$ were found in the TX-100insoluble membranes prepared from adult rat brain grey matter after the membranes were fractionated by sucrose gradient ultracentrifugation [72]. The lipid raft fractions were identified by the presence of flotillin. About $15 \%$ of total APP was associated with detergent-resistant membranes in the rat brain preparations suggesting that APP is present in the lipid rafts in vivo. Similarly, both $\mathrm{N}$ and C-terminal fragments of presenilin was also found in the lipid raft fractions. The $15 \%$ of APP found in the DIG fractions is consistent with $10 \%$ found in the lipid raft fractions of cultured cortical neurons in the TX-100-resistant membrane microdomains [68]. In this study, the authors also prepared detergent-resistant membranes and sucrose-gradient fractions from CHO cells stably expressing APP751. The identity of lipid rafts was confirmed by the presence of caveolin-1 as a
DIG marker. But unlike rat brain preparations, membrane microdomains from $\mathrm{CHO}$ cells contained only about $5 \%$ of APP indicating that in the brain a relatively larger fraction of APP is associated with lipid rafts. Interestingly, the percentage of $A \beta$ associated with lipid rafts in the rat brain also exceeds that found in the $\mathrm{CHO}$ cells. Thus the presence of APP, CTFs, A $\beta$ and presenilin components within the lipid raft fractions in the brain tissue in vivo strongly suggest that DIGs are the sites where amyloidogenic processing of APP occurs. But, in contrast to these results another study could not detect APP in SH-SY5Y cells in the TX-100-insoluble membrane microdomains after discontinuos sucrose-gradient ultracentrifugation, though they consistently found significant amounts of $A \beta$ by ELISA in detergent-insoluble fractions under similar condtions [73]. Experiments using 6E10immobilized columns also confirmed the presence of both $A \beta 40$ and $A \beta 42$ in the detergent-insoluble fractions from SH-SY5Y cells similar to that found in $\mathrm{CHO}$ cells and rat brain as described above [72]. Although this is quite intriguing, the absence of APP in the detergent-insoluble fractions in SH-SY5Y cells is consistent with another study as detailed above [69] and therefore association of APP in the lipid rafts may be cell type specific.

In a more recent study [74], AD brain hippocampal membranes contained most APP in the detergent-soluble microdomains and almost none in the insoluble DIG fractions. On the contrary, a significant amount of BACE1 was found in the detergent-insoluble lipid raft fractions. Based on these observations together with results from antibody co-patching experiments involving APP and BACE1, the authors even suggested that non-DIG domains of plasma membrane or internal membranes are the preferred sites for amyloidogenic processing of APP. Thus, BACE1 limited to lipid raft domains and APP to the non-lipid raft domains is suggested to be a mechanism to keep off the secretases from its substrate to prevent $A \beta$ generation [75]. To clarify whether inconsistency on the localization of APP in the lipid rafts is due to differences in the methodological approaches, Parkin et al. [76] examined APP in DIGs prepared from cerebral cortex under a range of protein to detergent ratios along with lipid raft markers. Various protein concentrations of $15,10,5$, and $2 \mathrm{mg} / \mathrm{ml}$ prepared from mouse cerebral cortex using $1 \% \mathrm{TX}-100$, did not reveal differences in the amounts of lipid raft markers such as flotillin and alkaline phosphatase. But the levels of APP in the lipid raft fractions could be detected only from the higher protein to detergent ratios and APP was completely absent from samples at lower ratios. Thus decreasing the protein/ detergent ratio completely excluded APP from lipid raft fractions suggesting that detection of APP in the lipid rafts depends on the protein to detergent ratio and therefore on the sensitivity of the assay. The absence of APP in the samples prepared at lower protein to detergent ratios was also confirmed in COS7 cells. Another interesting observation made in this study is that proteins in DIGs isolated from mouse cerebral cortex including APP was not soluble at both 4 and $37^{\circ} \mathrm{C}$. Lipid raft proteins are generally insoluble in TX-100 at $4^{\circ} \mathrm{C}$, but they are normally soluble at $37^{\circ} \mathrm{C}$ like most lipid raft marker proteins [21]. APP was also not soluble even at $37^{\circ} \mathrm{C}$ and therefore APP is considered to be an atypical lipid raft protein. This might explain the conflicting results on the localization of APP in the lipid 
rafts [68-73]. Even in those studies where APP was found in the lipid rafts, the amount of APP was also highly variable. As much as $15 \%$ of APP from total APP has been reported from rat brain preparations [72]. But Bouillot et al. reported only $1-5 \%$ of APP in the lipid rafts isolated from the embryonic cortical neurons [68] and only a minor part of total APP from APP transfected rat hippocampal neurons [44]. Thus, although many studies support possible amyloidogenic processing of APP based on the localization of APP in the lipid rafts, several studies question whether the lipid rafts are the primary sites for amyloidogenic processing because APP was present at the same levels as several non DIG proteins [74]. Membrane microdomains prepared from cells or tissues resistant to TX-100 extractions in cold and fractionated by sucrose gradient ultracentrifugation is considered a standard method to isolate lipid rafts [21]. The validity of this criterion has been verified by reconstitution experiments using purified GPI-anchored PLAP [77]. If a protein remains in the low buoyant fraction after detergent extraction, it is classified as a raft associated protein like flotillin, caveolin, alkaline phosphatase and $\mathrm{F} 3$ protein, which are all associated with lipid rafts and used as marker proteins. As discussed above, most studies used this standard protocol, yet there is not consistency as to whether APP, A $\beta$, CTFs and secretases are all present in the lipid rafts. Some proteins may be intrinsically insoluble in TX-100 and thus may be erroneously classified as lipid raft associated proteins. 1\% CHAPS is also used as a detergent to prepare lipid raft fractions. Using CHAPS as a detergent, we have consistently detected APP in the lipid raft fractions from CHO cells but not HEK293T cells $[78,79]$.

But what is even more intriguing is the localization of $A \beta$ in the absence of APP in the lighter lipid raft fractions [72, 73]. Both $A \beta 40$ and $A \beta 42$ were detected in the TX-100insoluble low-buoyant density membrane domains corresponding to lipid rafts. The lipid raft $A \beta$ which constituted more than half of total intracellular $A \beta$ as measured by ELISA was also confirmed by immunoprecipitation and immunoblotting, thus validating the findings by ELISA. A possible reason for the differential presence of A $\beta$ but not APP may be the intrinsic affinity of A $\beta$ with cholesterol [80] and ganglioside, GM1 [81-83], which form the core constituents of lipid rafts [21,84]. Cholesterol clearly influences A $\beta$ generation as discussed earlier [40-48]. Gangliosides are glycosphingolipids enriched in the neuronal plasma membrane. In the brain, GM1, GD1a, GD1b and GT1b types of gangliosides are present. Like cholesterol, exogenous addition of GM1 ganglioside increases amyloidogenic processing of APP and A $\beta$ generation in CHO cells, SH-SY5Y cells and primary neuronal cultures [85], though the mechanism remains unknown. One of the major sites of $A \beta$ generation in the neuron is ER and or Golgi compartments [86-89]. Because of its great affinity for cholesterol and gangliosides, $\mathrm{A} \beta$ produced at these sites could be a potential source for diffusion to the lipid rafts which are rich in cholesterol and gangliosides.

\section{Evidences from Proteins Involved in Lipid Raft Targeting of APP}

With inconsistent results on whether APP and secretases are localized to lipid rafts, many studies have also experi- mentally targeted APP and BACE1 to lipid rafts to study their influence on amyloidogenic processing of APP. GPIanchored proteins are a group of proteins attached to the membrane by a glycolipid anchor consisting of ethanolamine, mannose, glucosamine and phosphatidylinositol [90], which can be cleaved to free the protein by treatment with phosphatidylinositol-specific phospholipase C (PI-PLC). Lipid rafts are particularly enriched with post-translationally lipid modified proteins such as GPI-anchored proteins [9194] as well as doubly acylated [95] and palmitoylated proteins $[91,96]$. Therefore it is possible to target any protein for experimental studies specifically to lipid rafts by attaching either GPI-anchor, or introducing double acylation or palmitoylation. In one study [32], the transmembrane and cytosolic domains of BACE1 was replaced by GPI-anchor and expressed in SH-SY5Y cells. The majority of WTBACE1 was found in the TX-100 soluble membrane fractions suggesting that only a minor fraction of BACE1 is associated with lipid rafts, consistent with previous results $[33,76]$. However, addition of a GPI-anchor localized BACE1 almost exclusively to lipid raft fractions verified by flotillin enrichment similar to other GPI-anchored proteins. This led to $\sim 25$-fold increase in the levels of sAPP $\beta$ compared to WT-BACE1 expressing cells suggesting that $\beta$ site cleavage of APP was substantially increased after GPIaddition. $A \beta$ levels were undetectable in cells transfected with WT-BACE1, but cells expressing GPI-BACE1-anchor increased both $A \beta 40$ and $A \beta 42$ levels by as much as 11.6fold over control cells expressing WT-BACE1 which had undetectable levels of $A \beta$. The disruption of lipid rafts by cholesterol depletion led to substantial decrease in the levels of sAPP $\beta$ from both WT-BACE and GPI-BACE expressing cells suggesting that the increased amyloidogenic processing of APP by GPI-BACE is because of targeting of BACE to the lipid rafts which are presumably altered after cholesterol disruption. However, the significance of this data is slightly reduced because unfortunately this study did not attempt to localize whether APP is present in the lipid rafts. But considering that APP is absent in the lipid rafts in SH-SY5Y cells as previously suggested [69, 73], how could amyloidogenic processing of APP occur even if BACE is targeted to lipid rafts in the absence of its substrate, APP? This is an important question that should have been addressed in this study for a meaningful conclusion. In another more recent study, using S-palmitoylation as an alternative lipid raft targeting approach, Vetrivel and colleagues [97] demonstrated that BACE1 need not be associated with lipid rafts for amyloidogenic processing of APP in lipid rafts. In this study, alanine substitution of all four Cys residues, prevented palmitoylation-dependent targeting of BACE1 to lipid rafts in BACE1-deficient fibroblasts and N2a neuroblastoma cells, but surprisingly this did not have any effect on $\mathrm{A} \beta$ levels. This implies that BACE1 need not be associated with rafts per se to mediate beta-secretase cleavage of APP. However, one caveat of this conclusion is that both APP and BACE1 were studied under over expression paradigm; therefore, the overabundance of APP and BACE1 may have compensated from the lack of lipid raft targeting of BACE1.

In our own laboratory, we have consistently shown that low-density lipoprotein receptor-related protein (LRP) increases amyloidogenic processing of APP by promoting 
APP trafficking to lipid rafts [79]. To demonstrate this, HEK293T cells were co-transfected with Swe-APP751 and BACE1 together with or without EGFP-tagged soluble tail of LRP. CHAPS solubilized lysates were fractionated by sucrose-gradient ultracentrifugation and were subjected to immunoblotting. The results revealed that APP is not generally associated with lipid rafts in HEK293T cells or at least below the level of detection by immunoblots, but over expression of APP together with LRP-ST strongly increased lipid raft association of APP. LRP-ST itself was present in the lipid raft fractions suggesting that LRP-ST might be responsible for targeting APP to lipid rafts. LRP-ST also strongly increased BACE1 in lipid raft fractions. Similar results were also obtained in LRP-deficient 13-5-1 cells suggesting that LRP through its soluble tail promotes APP/LRP association with lipid rafts perhaps by facilitating the trafficking of APP/BACE1 to lipid rafts. Using another approach, we also demonstrated that endogenous LRP is necessary for delivery of APP to lipid rafts. CHO cells unlike HEK293T cells contain a considerable amount of APP in lipid rafts. Transfection of CHO-APP751 cells with LRP specific siRNAs resulted in the selective knockdown of LRP- $\beta$ chain, which in turn led to selective and marked reduction of APP-FL only in the lipid raft fractions relative to non-lipid raft fractions. This was also accompanied by more robust reductions in CTF levels in the lipid raft fractions and $\mathrm{A} \beta$ levels in the total lysates. Unlike WT-APP, the amount of Swe-APP in the lipid rafts is much less, however, LRP knockdown further reduced Swe-APP levels in the lipid rafts suggesting that LRP has similar lipid raft targeting effect on both WT and Swe APP, though the magnitudes are different. Thus these results not only support the idea that amyloidogenic processing of APP occurs in lipid rafts, but that LRP is responsible for normal delivery of APP to lipid rafts. Our finding that LRP-ST itself was associated with lipid rafts and promoted both APP and BACE1 targeting to lipid rafts also suggests that other yet unidentified proteins resident in lipid rafts may interact with LRP-ST and might influence APP/BACE1 association with lipid rafts. Therefore discovering novel LRP-ST-interacting proteins would provide further insights on the proteins or multiple protein complexes responsible for lipid raft targeting of APP/BACE1. The observation that internalization of APP increases amyloidogenic processing of APP is explained on the basis of the presence of tyrosine based endocytosis motif, NPxY in the cytoplasmic tails of both APP and LDL-R family members including LRP. This interpretation is supported by the observation that mutation of NPxY motif decreases $A \beta$ production. Having identified the last 37 amino acids (LRP-C37) of LRP, which retains the conserved dileucine motif but lacks the NPxY motifs to be sufficient to robustly promote $A \beta$ generation, we used LRPC37 domain as bait in a yeast 2-hybrid screen to identify potential effectors of APP processing and trafficking [98]. From this screen, we identified RanBP9 as a novel LRPC37-interacting protein. RanBP9 acts as a scaffolding molecule for a variety of membrane receptors bridging their cytoplasmic tails with the intracellular targets. Our subsequent

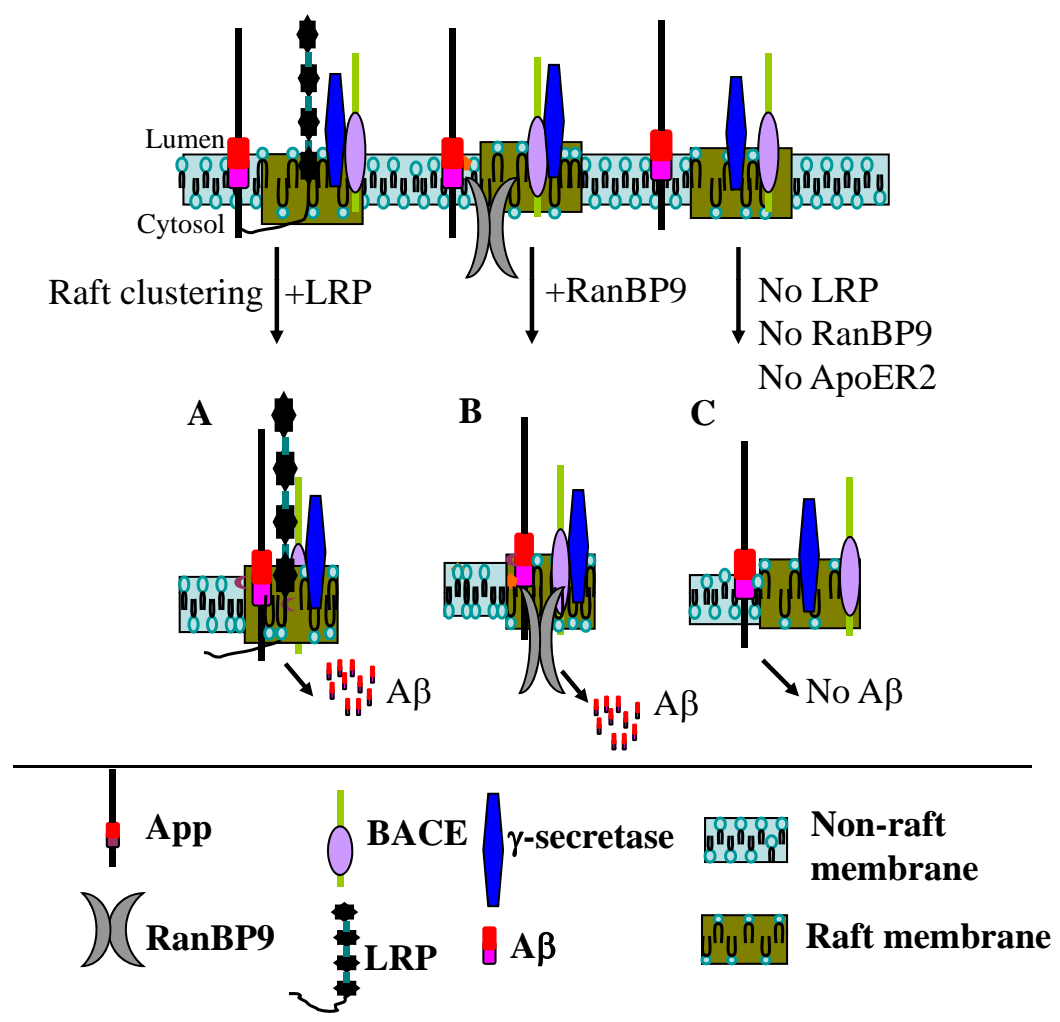

Fig. (2). A schematic of protein mediated lipid raft targeting of APP. Proteins such as LRP and ApoER2 are receptors localized to the plasma membrane and RanBP9 is localized to the inner leaflet of plasma membrane as well as cytosol. All three proteins bind and deliver APP to the lipid rafts following raft clustering. (A) Over expression of LRP or ApoER2 leads to delivery of non-raft associated APP to lipid rafts. (B) Over expression of RanBP9 also leads to enrichment of APP in the lipid rafts. (C) By selectively knocking down the protein levels of LRP, RanBP9 or ApoER2, it is possible to prevent lipid raft association and amyloidogenic processing of APP as has been demonstrated for LRP. This could be an alternative and viable therapeutic option for AD. 
experiments showed that RanBP9 not only binds LRP, but also APP and BACE1 in both cell cultures and in vivo in mouse brains. Interestingly, like LRP-ST and LRP-C37, RanBP9 robustly increased $A \beta$ secretion in a variety of cell lines. It is even more important that like LRP, RanBP9 also targeted APP to lipid raft domains as demonstrated by CHAPS-insoluble membrane preparations followed by discontinuous sucrose density gradient fractionations from CHO cells. The levels of APP, $\beta$ CTFs and sAPP $\beta$ were enriched in lipid raft fractions from $\mathrm{CHO}$ cells transfected with APP and RanBP9 compared to fractions from $\mathrm{CHO}$ cells expressing only APP, but flotillin remained unchanged from both groups [78]. Similar to LRP, APP and BACE1, RanBP9 was also enriched in lipid raft fractions consistent with its role in targeting APP to lipid rafts. It is possible that the interaction of RanBP9 with LRP, BACE1 and APP may be independent of each other. We also demonstrated that LRP not only enhances APP-RanBP9 interaction but at the same time RanBP9 also reinforces both APP-BACE1 and APP-LRP complexes suggesting that RanBP9 positively regulates the physical association of LRP, APP and BACE1. These data taken together indicate that RanBP9 in concert with LRP promotes the association of APP with lipid rafts, where BACE1-mediated $\beta$-cleavage and $A \beta$ generation occurs. In this scenario whether LRP recruits RanBP9 or vice versa to target APP to lipid raft microdomains needs to be investigated further. Because RanBP9 has multiple protein-protein interacting domains including proline rich domain (PRD), SPRY and LisH domains, RanBP9 may be able to scaffold not only LRP but also BACE1 and APP simultaneously forming high molecular weight complexes to promote their movement to lipid rafts. Over all, we have clearly demonstrated that both LRP and RanBP9 increases amyloidogenic processing of APP in a similar manner perhaps by targeting APP and/or BACE1 to lipid raft microdomains (Fig. 2).

Support for protein mediated delivery of APP to lipid raft microdomains also comes from another study involving ApoER2, another member of the low density lipoprotein receptor (LDL-R) highly expressed in the brain [99]. To assess the effect of ApoER2 on APP trafficking and processing, CHO cells lacking LRP1 were transfected with human cDNA for ApoER2. The results from flow immunocytometry revealed that cell surface levels of APP were increased in cells expressing ApoER2 relative to control cells suggesting that ApoER2 regulates sub cellular distribution of APP. Using myc-tagged APP695 and HA-tagged ApoER2, APP was shown to physically bind to ApoER2 by co-immunoprecipitation assay in $\mathrm{N} 2$ a cells lacking detectable levels of any member of the LDL-R family. Like LRP and RanBP9, ApoER2 expression also increased generation and secretion of $A \beta$. To verify if the increased amyloidogenesis is due to lipid raft targeting of APP, Lubrol-insoluble membrane prepartions at $4^{\circ} \mathrm{C}$ isolated from APP- or APPApoER2 expressing $\mathrm{CHO}$ cells were fractionated by sucrosegradient ultracentrifugation and analyzed by immunoblots. ApoER2 expression increased APP in the lipid raft membrane domains by 2.2-fold compared to control cells, but the levels of lipid raft resident protein, caveolin-1 used as control was unaltered suggesting that ApoER2 specifically targets APP to lipid rafts. However, ApoER2 expression markedly reduced CTF levels in the lipid raft microdomains unlike LRP and RanBP9 which strongly and consistently increased CTF levels in multiple experiments in the same light weight fractions [78, 79] suggesting that the mechanism of action of ApoER2 on lipid raft targeting of APP may be entirely different from that of LRP and RanBP9. The difference also lies in the fact that while LRP and RanBP9 increased APP endocytosis, ApoER2 expression increased APP association to lipid rafts by decreasing the rate of APP endocytosis. But this does not contradict the RanBP9/LRP mediated increased endocytosis as the basis for increased amyloidogenic processing because lipid rafts are located not only in the plasma membrane but also in various intracellular compartments such as early endosomes, TGN and early recycling vesicles. It is not clear if the detergent used in this study, Lubrol versus CHAPS was responsible for the difference. But the similarity lies in the fact that all three proteins LRP, RanBP9 and ApoER2 physically interact with APP and therefore their effect on APP targeting to lipid rafts may be direct (Fig. 2).

\section{Targeting Lipid Rafts for Therapeutic Intervention in AD}

The currently available drugs for $\mathrm{AD}$ do not reverse the clinical course and therefore novel mechanism based therapies are urgently required. The major focus of research in recent years has been to find ways to reduce the level of the disease causing/modifying agent, $A \beta$, in the brain as a therapeutic strategy for AD. At present there are three general approaches to obstructing the $A \beta$ cascade: (1) inhibit the production of $A \beta ;(2)$ block the aggregation and toxicity of $A \beta$; and (3) stimulate the degradation and removal of $A \beta$. Among these three approaches, inhibiting the production of $\mathrm{A} \beta$ is the widely pursued method because the enzymes involved in the generation of $\mathrm{A} \beta$ has been identified and well characterized. Inhibiting the $\beta$ - and $\gamma$-secretases is an obvious therapeutic strategy that many investigators both in academia and industry are actively pursuing. But since both $\beta$ and $\gamma$-secretases are involved in the cleavage of proteins essential in many cellular processes, a legitimate concern has been raised that the secretase inhibitors may be associated with many adverse and potentially intolerable side effects. This is probably the reason for early withdrawal of some of the $\gamma$-secretase inhibitors from clinical trials. Therefore alternative approaches are very important at this juncture.

As detailed above the secretases involved in APP processing have been localized to the lipid rafts. If secretases are selectively inhibited only in the lipid rafts, where amyloidogenic processing of APP presumably occur, sparing secretases localized elsewhere vital for several other functions, the adverse effects associated with global inhibition of secretases can be avoided. In fact, recently Rajendran et al. [100] used this strategy and successfully demonstrated that APP processing and $A \beta$ generation can be significantly reduced both in cell cultures and in transgenic mouse models. Rajendran and colleagues cleverly added a sterol moiety as a membrane anchor to a known $\beta$-secretase transition-state inhibitor and targeted the drug to endosomes/lipid rafts where the enzyme is active. By doing this, the inhibitor was not only localized to the endosomes/lipid rafts, but also increased the inhibitory potential of the drug, which in turn led to significant 
reduction in $\mathrm{A} \beta$ generation. This confirms the importance of endosomes/lipid rafts for $A \beta$ generation and thus raises the hopes of new approach to influence the progression of $\mathrm{AD}$ based on lipid rafts.

Further, those proteins which deliver APP to the lipid rafts such as LRP [79], RanBP9 [78] and ApoER2 [99] can be excellent therapeutic targets in Alzheimer's disease. Over expression of these proteins clearly demonstrated the lipid raft targeting of APP and increased amyloidogenesis. Conversely, specific knockdown of LRP with siRNAs reversed the amyloidogenic processing by inhibiting transport of APP to lipid rafts suggesting that by modulating LRP expression, it is possible to limit the entry of APP to lipid rafts thereby reducing A $\beta$ generation [79]. Similar approach may also work for both RanBP9 and ApoER2, though it is not yet clear whether reducing these protein levels would also reduce APP entry into lipid raft microdomains. To date, LRP, RanBP9 and ApoER2 are the only proteins identified that is critical for association of APP to lipid rafts. Identification of small molecule drugs that can potentially block LRP, RanBP9 or ApoER2 dependent delivery of APP to lipid rafts would be predicted to diminish $A \beta$ generation (Fig. 2). Since this approach does not involve inhibition of secretases directly, the unintended side effects associated with inhibition of secretases may be completely avoided.

\section{ACKNOWLEDGEMENTS}

We are highly grateful to Prof. Edward Koo, University of California, San Diego for his invaluable suggestions. MKL was supported in part by NIH/NIA grant \# 5R03AG032064-02, and DEK was supported in part by the American Health Assistance Foundation grant \# A2007-05 and WCU grant from KOSEF.

\section{REFERENCES}

[1] Alzheimer's association. 2009 Alzheimer's disease facts and figures. Alzheimer's Dement 2009; 5: 234-70.

[2] Graeber MB, Kosel S, Egensperger R, et al. Rediscovery of the case described by Alois Alzheimer in 1911: historical, histological, and molecular genetic analysis. Neurogenetics 1997; 1: 73-80.

[3] Selkoe DJ. Alzheimer's disease: genes, protein, and therapy. Physiolog Rev 2001; 81: 741-66.

[4] Jacobsen KT, Iverfeldt K. Amyloid precursor protein and its homologues: a family of proteolysis-dependent receptors. Cell Mol Life Sci 2009; 66: 2299-318.

[5] Kang J, Lemaire HG, Unterbeck A, et al. The precursor of Alzheimer's disease amyloid A4 protein resembles a cell-surface receptor. Nature 1987; 325: 733-6.

[6] Zheng H, Jiang M, Trumbauer ME, et al. Beta-Amyloid precursor protein-deficient mice show reactive gliosis and decreased locomotor activity. Cell 1995; 81: 525-31.

[7] Von Koch CS, Zheng H, Chen H, et al. Generation of APLP2 KO mice and early postnatal lethality in APLP2/APP double KO mice. Neurobiol Aging 1997; 18: 661-9.

[8] Heber S, Herms J, Gajic V, et al. Mice with combined gene knockouts reveal essential and partially redundant functions of amyloid precursor protein family members. J Neurosci 2000; 20: 7951-63.

[9] Selkoe DJ, Podlisny MB, Joachim CL, et al. Beta-amyloid precursor protein of Alzheimer disease occurs as 110- to 135kilodalton membrane-associated proteins in neural and nonneural tissues. Proc Natl Acad Sci USA 1988; 85: 7341-45.

[10] Hung AY, Selkoe DJ. Selective ectodomain phosphorylation and regulated cleavage of beta-amyloid precursor protein. EMBO J 1994; 13: 534-42.
[11] Oltersdorf T, Ward PJ, Henrickson T, et al. The Alzheimer amyloid precursor protein. Identification of a stable intermediate in the biosynthetic/degradative pathway. J Biol Chem 1990; 265: 4492-7.

[12] Walter J, Capell A, Hung AY, et al. Ectodomain phosphorylation of beta-amyloid precursor protein at two distinct cellular locations. J Biol Chem 1997; 272: 1896-903.

[13] Haass C, Hung AY, Selkoe DJ. Processing of beta-amyloid precursor protein in microglia and astrocytes favors an internal localization over constitutive secretion. J Neurosci 1991; 11: 378393.

[14] Sisodia SS. Beta-amyloid precursor protein cleavage by a membrane-bound protease. Proc Natl Acad Sci USA 1992; 89: 6075-9.

[15] Kojro E, Fahrenholz F. The non-amyloidogenic pathway: structure and function of alpha secretases. Subcell Biochem 2005; 38: 10527.

[16] Cole SL, Vassar R. The role of amyloid precursor protein processing by BACE1, the beta secretase in Alzheimer's disease pathogenesis. J Biol Chem 2008; 283: 29621-5.

[17] De Strooper B, Annaert W. Proteolytic processing and cell biological functions of the amyloid precursor protein. J Cell Sci 2000; 113: 1857-70.

[18] Koo EH, Squazzo SL. Evidence that production and release of amyloid beta-protein involves the endocytic pathway. J Biol Chem 1994; 269: 17386-9.

[19] Perez RG, Soriano S, Hayes JD, et al. Mutagenesis identifies new signals for beta-amyloid precursor protein for endocytosis,turnover, and the generation of secreted fragments including Abeta42. J Biol Chem 1999; 274: 18851-6.

[20] Simons K, Ikonen E. Functional rafts in cell membranes. Nature 1997; 387: 569-72.

[21] Brown DA, Rose JK. Sorting of GPI-anchored proteins to glycolipid-enriched membrane subdomains during transport to the apical cell surface. Cell 1992; 68: 533-44.

[22] Parton RG, Simons K. Digging into caveolae. Science 1995; 269: 1398-9.

[23] Skibbens JE, Roth MG, Matlin KS. Differential extractability of influenza virus hemagglutinin during intracellular transport in polarized epithelial cells and nonpolar fibroblasts. J Cell Biol 1989; 108: 821-32.

[24] Sargiacomo M, Sudol M, Tang Z, Lisanti MP. Signal transducing molecules and glycosyl-phosphatidyl inositol-linked proteins form a caveolin-rich insoluble complex in MDCK cells. J Cell Biol 1993; 122: 789- 807

[25] Danielsen EM. Involvement of detergent-insoluble complexes in the intracellular transport of intestinal brush border enzymes. Biochemistry 1995; 34: 1596-605.

[26] Fiedler K, Kobayashi T, Kurzchalia TV, Simons K. Glycosphingolipid-enriched, detergent-insoluble complexes in protein sorting in epithelial cells. Biochemistry 1993; 32: 6365-73.

[27] Brown DA, London E. Structure of detergent-resistant membrane domains: does phase separation occur in biological membranes? Biochem Biophys Res Commun 1997; 240: 1-7.

[28] Shaw AS. Lipid rafts: Now you can see them, now you don't. Nat Immunol 2006; 7: 1139-42.

[29] Lai EC. Lipid rafts make for slippery platform. J Cell Biol 2003 162: 365-70

[30] Lee SJ, Liyanage U, Bickel PE, Xia W, Lansbury PT Jr, Kosik KS. A detergent-insoluble membrane compartment contains A beta in vivo. Nat Med 1998; 4: 730-4.

[31] Kawarabayashi T, Shoji M, Younkin LH, et al. Dimeric amyloid beta protein rapidly accumulates in lipid rafts followed by apolipoprotein E and phosphorylated tau accumulation in the Tg2576 mouse model of Alzheimer's disease. J Neurosci 2004; 24 : 3801-9.

[32] Cordy JM, Hussain I, Dingwall C, Hooper NM, Turner AJ. Exclusively targeting beta-secretase to lipid rafts by GPI-anchor addition up-regulates beta-site processing of the amyloid precursor protein. Proc Natl Acad Sci USA 2003; 100: 11735-40.

[33] Riddell DR, Christie G, Hussain I, Dingwall C. Compartmentalization of beta-secretase [Asp2] into low- buoyant density, noncaveolar lipid rafts. Curr Biol 2001; 11: 1288-93.

[34] Vetrivel KS, Cheng H, Kim SH, et al. Spatial segregation of gamma-secretase and substrates in distinct membrane domains. J Biol Chem 2005; 280: 25892-900. 
[35] Parkin ET, Turner AJ, Hooper NM. Detergent solubility and processing of the familial Alzheimer's disease- related presenilin proteins. Biochem Soc Trans 1998; 26: S241.

[36] Wahrle S, Das P, Nyborg AC, et al. Cholesterol-dependent gammasecretase activity in buoyant cholesterol- rich membrane microdomains. Neurobiol Dis 2002; 9: 11-23.

[37] Hung, YH, Robb EL, Volitakis I. et al. Copper sensitively induces presenilin 1 lipid raft localization with increased amyloidogenic processing of the amyloid precursor protein. Alzheimers Dement 2006; 2: S58-65.

[38] Vetrivel KS, Cheng H, Lin W, et al. Association of gammasecretase with lipid rafts in post-Golgi and endosome membranes. $\mathrm{J}$ Biol Chem 2004; 279: 44945-54.

[39] Urano $\mathrm{Y}$, Hayashi I, Isoo N, et al. Association of active gammasecretase complex with lipid rafts. J Lipid Res 2005; 46: 904-12.

[40] Corder EH, Saunders AM, Strittmatter WJ, et al. Gene dose of apolipoprotein E type 4 allele and the risk of Alzheimer's disease in late onset families. Science 1993; 261: 921-3.

[41] Strittmatter WJ, Saunders AM, Schmechel D, et al. Apolipoprotein E: high-avidity binding to beta- amyloid and increased frequency of type 4 allele in late-onset familial Alzheimer disease. Proc Natl Acad Sci USA 1993; 90: 1977-81.

[42] Jick H, Zornberg GL, Jick SS, Seshadri S, Drachman DA. Statins and the risk of dementia. Lancet 2000; 356: 1627-31.

[43] Kojro E, Gimpl G, Lammich S, Marz W, Fahrenholz F. Low cholesterol stimulates the nonamyloidogenic pathway by its effect on the alpha-secretase ADAM 10. Proc Natl Acad Sci USA 2001; 98: 5815-20.

[44] Simons M, Keller P, De Strooper B, Beyreuther K, Dotti CG, Simons K. Cholesterol depletion inhibits the generation of betaamyloid in hippocampal neurons. Proc Natl Acad Sci USA 1998; 95: 6460-4.

[45] Pappolla MA, Bryant-Thomas TK, Herbert T, et al. Hypercholesterolemia is an early risk factor for the development of Alzheimer amyloid pathology. Neurology 2003; 61: 199-205.

[46] Kivipelto M, Helkala EL, Laakso MP, et al. Midlife vascular risk factors and Alzheimer's disease in later life: longitudinal, population based study. BMJ 2001; 322:1447-51.

[47] Fassbender K, Simons M, Bergmann C, et al. Simvastatin strongly reduces levels of Alzheimer's disease beta-amyloid peptides Abeta 42 and Abeta 40 in vitro and in vivo. Proc Natl Acad Sci USA 2001; 98: 5856-61.

[48] Hutter-Paier B, Huttunen HJ, Puglielli L, et al. The ACAT inhibitor CP-113,818 markedly reduces amyloid pathology in a mouse model of Alzheimer's disease. Neuron 2004; 44: 227-38.

[49] Meske V, Albert F, Richter D, Schwarze J, Ohm TG. Blockade of HMG-CoA reductase activity causes changes in microtubulestabilizing protein tau via suppression of geranylgeranylpyrophosphate formation: implications for Alzheimer's disease. Eur J Neurosci 2003; 17: 93-102.

[50] Green RC, McNagny SE, Jayakumar P, Cupples LA, Benke K, Farrer LA. Statin use and the risk of Alzheimer's disease: The MIRAGE Study. Alzheimers Dement 2006; 2: 96-103.

[51] Ledesma MD, Abad-Rodriguez J, Galvan C, et al. Raft disorganization leads to reduced plasmin activity in Alzheimer's disease brains. EMBO Rep 2003; 4: 1190-6.

[52] Howland DS, Trusko SP, Savage MJ, et al. Modulation of secreted beta-amyloid precursor protein and amyloid beta-peptide in brain by cholesterol. J Biol Chem 1998; 273: 16576-82.

[53] Park IH, Hwang EM, Hong HS, et al. Lovastatin enhances Abeta production and senile plaque deposition in female Tg2576 mice. Neurobiol Aging 2003; 24: 637-43.

[54] Won J-S, Im YB, Khan M, Contreras M, Singh AK, Singh I. Lovastatin inhibits amyloid precursor protein [APP] beta-cleavage through reduction of APP distribution in Lubrol WX extractable low density lipid rafts. J Neurochem 2008; 105: 1536-49.

[55] Refolo LM, Sambamurti K, Efthimiopoulos S, Pappolla MA, Robakis NK. Evidence that secretase cleavage of cell surface Alzheimer amyloid precursor occurs after normal endocytic internalization. J Neurosci Res 1995; 40: 694-706.

[56] Soriano S, Chyung AS, Chen X, Stokin GB, Lee VM, Koo EH. Expression of beta-amyloid precursor protein-CD3 gamma chimeras to demonstrate the selective generation of amyloid beta(1$40)$ and amyloid beta(1-42) peptides within secretory and endocytic compartments. J Biol Chem 1999; 274: 32295-300.
[57] Walter J, Fluhrer R, Hartung B, et al. Phosphorylation regulates intracellular trafficking of beta-secretase. J Biol Chem 2001; 276: 14634-41.

[58] Bamberger ME, Harris ME, McDonald DR, Husemann J, Landreth GE. A cell surface receptor complex for fibrillar beta-amyloid mediates microglial activation. J Neurosci 2003; 23: 2665-74.

[59] Grbovic OM, Mathews PM, Jiang Y, et al. Rab5-stimulated upregulation of the endocytic pathway increases intracellular betacleaved amyloid precursor protein carboxyl-terminal fragment levels and Abeta production. J Biol Chem 2003; 278: 31261-8.

[60] Cataldo AM, Petanceska S, Terio NB, et al. Abeta localization in abnormal endosomes: association with earliest Abeta elevations in AD and Down syndrome. Neurobiol Aging 2004; 25: 1263-72.

[61] Cole SL, Vassar R. The Alzheimer's disease beta-secretase enzyme, BACE1. Mol Neurodegener 2007; 2: 22.

[62] Vassar R, Bennett BD, Babu-Khan S, et al. Beta-secretase cleavage of Alzheimer's amyloid precursor protein by the transmembrane aspartic protease BACE. Science 1999; 286: 735-41.

[63] Ehehalt R, Keller P, Haass C, Thiele C, Simons K. Amyloidogenic processing of the Alzheimer beta- amyloid precursor protein depends on lipid rafts. J Cell Biol 2003; 160: 113-23.

[64] Prior IA, Harding A, Yan J, Sluimer J, Parton RG, Hancock JF. GTP-dependent segregation of H-ras from lipid rafts is required for biological activity. Nat Cell Biol 2001; 3: 368-75.

[65] Harder T, Scheiffele P, Verkade P, Simons K. Lipid domain structure of the plasma membrane revealed by patching of membrane components. J Cell Biol 1998; 141: 929-42.

[66] Lanzetti L, Rybin V, Malabarba MG, et al. The Eps8 protein coordinates EGF receptor signaling through Rac and trafficking through Rab5. Nature 2000; 408: 374-7.

[67] Damke H, Baba T, Warnock DE, Schmid SL. Induction of mutant dynamin specifically blocks endocytic coated vesicle formation. J Cell Biol 1994; 127: 915-34.

[68] Bouillot C, Prochiantz A, Rougon G, Allinquant B. Axonal amyloid precursor protein expressed by neurons in vitro is present in a membrane fraction with caveolae-like properties. J Biol Chem 1996; 271: 7640-4.

[69] Parkin ET, Hussain I, Turner AJ, Hooper NM. The amyloid precursor protein is not enriched in caveolae- like, detergentinsoluble membrane microdomains. J Neurochem 1997; 69: 217988.

[70] Ikezu T, Trapp BD, Song KS, Schlegel A, Lisanti MP, Okamoto T. Caveolae, plasma membrane microdomains for alpha-secretasemediated processing of the amyloid precursor protein. J Biol Chem 1998; 273:10485-95.

[71] Kang MJ, Chung YH, Hwang CI, et al. Caveolin-1 upregulation in senescent neurons alters amyloid precursor protein processing. Exp Mol Med 2006; 38: 126-33.

[72] Lee SJ, Liyanage U, Bickel PE, Xia W, Lansbury PT, Jr., Kosik KS. A detergent-insoluble membrane compartment contains $A \beta$ in vivo. Nat Med 1998; 4: 730-34.

[73] Morishima-Kawashima M, Ihara Y. The presence of amyloid betaprotein in the detergent-insoluble membrane compartment of human neuroblastoma cells. Biochemistry 1998; 37: 15247-53.

[74] Abad-Rodriguez J, Ledesma MD, Craessaerts K, et al. Neuronal membrane cholesterol loss enhances amyloid peptide generation. J Cell Biol 2004; 167: 953-60.

[75] Koether C, Haass C. A lipid raft boundary separates APP and secretases and limits amyloid $\beta$ - peptide generation. J Cell Biol 2004; 167: 809-12.

[76] Parkin ET, Turner AJ, Hooper NM. Amyloid precursor protein, although partially detergent-insoluble in mouse cerebral cortex, behaves as an atypical lipid raft protein. Biochem J 1999; 344: 2330 .

[77] Schroeder R, London E, Brown D. Interactions between saturated acyl chains confer detergent resistance on lipids and glycosylphosphatidylinositol (GPI)-anchored proteins: GPIanchored proteins in liposomes and cells show similar behavior. Proc Natl Acad Sci USA 1994; 91: 12130-4.

[78] Lakshmana MK, Yoon IS, Chen E, Bianchi E, Koo EH, Kang DE. Novel role of RanBP9 in BACE1 processing of amyloid precursor protein and amyloid beta peptide generation. J Biol Chem 2009; 284: 11863-72.

[79] Yoon IS, Chen E, Busse T, et al. Low-density lipoprotein receptorrelated protein promotes amyloid precursor protein trafficking to lipid rafts in the endocytic pathway. FASEB J 2007; 21: 2742-52. 
[80] Avdulov NA, Chochina SV, Igbavboa U, Warden CS, Vassiliev AV, Wood WG. Lipid binding to amyloid beta-peptide aggregates: preferential binding of cholesterol as compared with phosphatidylcholine and fatty acids. J Neurochem 1997; 69: 174652.

[81] Choo-Smith LP, Garzon-Rodriguez W, Glabe CG, Surewicz WK. Acceleration of amyloid fibril formation by specific binding of Abeta-[1-40] peptide to ganglioside-containing membrane vesicles. J Biol Chem 1997; 272: 22987-90.

[82] Choo-Smith LP, Surewicz WK. The interaction between Alzheimer amyloid beta [1-40] peptide and ganglioside GM1-containing membranes. FEBS Lett 1997; 402: 95-8.

[83] Matsuzaki K, Horikiri C. Interactions of amyloid beta-peptide (140) with ganglioside-containing membranes. Biochemistry 1999; 38: 4137-42.

[84] Vey M, Pilkuhn S, Wille H, et al. Subcellular colocalization of the cellular and scrapie prion proteins in caveolae-like membranous domains. Proc Natl Acad Sci USA 1996; 93: 14945-9.

[85] Zha Q, Ruan Y, Hartmann T, Beyreuther K, Zhang D. GM1 ganglioside regulates the proteolysis of amyloid precursor protein. Mol Psychiatry 2004; 9: 946-52.

[86] Hartmann T, Bieger SC, Bruhl B, et al. Distinct sites of intracellular production for Alzheimer's disease A beta40/42 amyloid peptides. Nat Med 1997; 3: 1016-20.

[87] Cook DG, Forman MS, Sung JC, et al. Alzheimer's A beta (1-42) is generated in the endoplasmic reticulum/intermediate compartment of NT2N cells. Nat Med 1997; 3: 1021-3.

[88] Wild-Bode C, Yamazaki T, Capell A, et al. Intracellular generation and accumulation of amyloid beta peptide terminating at amino acid 42. J Biol Chem 1997; 272: 16085-8.

[89] Xu H, Sweeney D, Wang R, et al. Generation of Alzheimer betaamyloid protein in the trans-Golgi network in the apparent absence of vesicle formation. Proc Natl Acad Sci USA 1997; 94: 3748-52.
[90] McConville MJ, Ferguson MA. The structure, biosynthesis and function of glycosylated phosphatidylinositols in the parasitic protozoa and higher eukaryotes. Biochem J 1993; 294: 305-24.

[91] Brown DA, London E. Functions of lipid rafts in biological membranes. Annu Rev Cell Dev Biol 1998; 14: 111-36.

[92] Hooper NM. Detergent-insoluble glycosphingolipid/cholesterolrich membrane domains, lipid rafts and caveolae (review). Mol Membr Biol 1999; 16: 145-56.

[93] Harder T, Scheiffele P, Verkade P, Simons K. Lipid domain structure of $t$ he plasma membrane revealed by patching of membrane components. J Cell Biol 1998; 141: 929-42.

[94] Mayor S, Rothberg KG, Maxfield FR. Sequestration of GPIanchored proteins in caveolae triggered by cross-linking. Science 1994; 264: 1948-51.

[95] Resh MD. Fatty acylation of proteins: new insights in to membrane targeting of myristoylated and palmitoylated proteins. Biochim Biophys Acta 1999; 1451: 1-16.

[96] Rietveld A, Neutz S, Simons K, Eaton S. Association of sterol- and glycosylphosphatidyl inositol-linked proteins with Drosophila raft lipid microdomains. J Biol Chem 1999 274: 12049-54.

[97] Vetrivel KS, Meckler X, Chen Y, et al. Alzheimer disease Abeta production in the absence of S- palmitoylation-dependent targeting of BACE1 to lipid rafts. J Biol Chem 2009; 284: 3793-803.

[98] Lakshmana MK, Chen E, Yoon IS, Kang DE. C-terminal 37 residues of LRP promote the amyloidogenic processing of APP independent of FE65. J Cell Mol Med 2008; 12: 2665-74.

[99] Fuentealba RA, Barria MI, Lee J, et al. ApoER2 expression increases Abeta production while decreasing amyloid precursor protein (APP) endocytosis: Possible role in the partitioning of APP into lipid rafts and in the regulation of gamma-secretase activity. Mol Neurodegener 2007; 2: 14.

[100] Rajendran L, Schneider A, Schlechtingen G, et al. Efficient inhibition of the Alzheimer's disease beta- secretase by membrane targeting. Science 2008; 320: 520-3.

(C) Lakshmana et al.; Licensee Bentham Open.

This is an open access article licensed under the terms of the Creative Commons Attribution Non-Commercial License (http://creativecommons.org/licenses/bync/3.0/), which permits unrestricted, non-commercial use, distribution and reproduction in any medium, provided the work is properly cited. 